\title{
Specific features of noise spreading from motor transport in the urban environment
}

\author{
Irina Margolina * and Denis Veselov \\ Faculty of Geography, L omonosov M oscow State U niversity, Leninskie gory, 119991, M oscow, \\ Russian Federation
}

\begin{abstract}
The work is devoted to the study of the features of Spatiotemporal changes in the noise level from vehicles and is based on field measurements carried out in the autumn-spring, summer and winter seasons. The measurements were carried out on the territory of the administrative district of M oscow at 861 points during the evening rush hour. For the analysis of space-time series, a cartographic research method was used using the QuantumGIS and A rcGis programs. The series of maps created in this work demonstrates the dependence of the noise level in the urban area on the traffic load, the type of urban planning, and seasonality (the presence of snow cover and the vegetation state of plant complexes). Zoning of the territory by the noise influence makes it possible to identify zones with a critical and high noise level (above the PEL standard), as well as areas with an insignificant and medium noise level (below the PEL).
\end{abstract}

\section{Introduction}

The level of urbanization in the world is increasing, leading to an increase in the noise level exposure for the population. The main source of noise in cities is road transport, which significantly increases the noise impact on residential areas located near highways [1]. Currently, the study of the spread of noise is becoming more and more relevant in connection with the implementation of measures for the renovation of dilapidated housing in many large urban agglomerations [2].

To study the spread of the noise level in the residential area, an administrative district of Moscow was selected, which is surrounded by major highways and having various types of urban planning: quarters with a preserved five-storey housing stock, renovation areas, highrise buildings, and green areas.

The Spatio-temporal dynamics of the noise level depends on many factors, including the force of the noise source and the noise-absorbing properties of the environment, which have seasonal variability. The work identified three main seasons: summer (with foliage of plant complexes), winter (with established snow cover), and autumn-spring (with a complete

*Corresponding author: irina-mgu@ mail.ru 
absence of foliage and snow cover). It is assumed that within one season, climatic parameters do not have significant changes that affect the distribution of noise.

\section{Materials and research methods}

The study of the features of Spatio-temporal changes in the noise level was carried out on the example of the territory of the Vernadsky Prospekt administrative district of the city aof Moscow with an area of $4.6 \mathrm{~km}^{2}$, with a population of 64 thousand people. The territory of the district is characterized by insignificant elevation changes with a slight decrease to the south-west towards the Smorodinka river valley, the presence of green areas (the park of the 50th anniversary of October, and the park of the Olympic village), various types of urban planning. The investigated territory is surrounded by highways of Leninsky Prospekt, Michurinsky Prospekt, and Vernadsky Prospekt with 6-8 lanes, as well as motorways of Lobachevsky, Obruchev, Udaltsov, Koshtoyants streets with 4-6 lanes; the district is pierced by intra-quarter roads with two-lane traffic.

To carry out the measurements, the territory of the district was covered with a regular grid with a step of 100 meters in the corners of which the noise level was measured according to the seasons of the year (November 2017 - November 2018). In cases where the calculated measurement of location fell into an inaccessible area (water body, building, road, etc.), the measurement was carried out at the closest available point. 861 points were set in which the values of the noise level were recorded for three seasons (summer, winter, spring-autumn). The Spatio-temporal studies of the noise level in the city are focused on the analysis of noise impact from the main noise source - vehicles [1], therefore, for further data comparison, all measurements were carried out in the middle of the working week (Wednesday, Thursday) during the evening rush hour from 18.30 to 20.00 , corresponding to the highest intensity of traffic flows in all studied seasons of the year.

The noise was measured with a second class sound level meter (AKTAKOM ATT-9000). To exclude random noise impacts typical for residential areas (barking, creaking, talking, etc.), the arithmetic mean value was calculated for three consecutive readings every 1-2 minutes. To assess the magnitude of the impact, the standards for the territories adjacent to the residential areas in the settlements was used [3].

The information obtained as a result of measurements was processed by the methods of geoinformation mapping and modeling in the programs Quantum GIS and ArcGIS [4], which made it possible to carry out a Spatio-temporal analysis of changes in the noise level based on instrumental data obtained from a continuous sample.

\section{Analysis of research results}

Based on the limiting values of the PEL standards for the territories adjacent to the residential area [3], four gradations of values were identified [Table], from which it can be seen that there is an annual dynamics of noise levels by seasons, with the highest values in the springautumn period, and with the lowest values in summer. Since the studies were carried out in the daytime, then the loads "Insignificant" and "Medium" correspond to the permissible noise levels, while the "Strong" and "Critical" ones correspond to the values above the established standards.

For an average annual assessment of noise pollution, the value of the averaged impact was calculated, taking into account the weight coefficients of the duration of the seasons. For this, according to the climatic and phenological data [5], the ratio of the leafing period and the established snow cover for the Moscow region was determined. 
Table. Dynamics of noise influence level by seasons.

\begin{tabular}{|l|l|c|c|c|c|}
\hline \multirow{2}{*}{$\begin{array}{l}\text { Noise } \\
\text { gradation, } \\
\text { in dB A. }\end{array}$} & N oise influence & \multicolumn{4}{|c|}{ A reas with different noise influence by season, in \% } \\
\cline { 3 - 6 } & & $\begin{array}{c}\text { A utumn/ } \\
\text { Spring }\end{array}$ & W inter & Summer & A verage \\
\hline$<45$ & Insignificant & 15 & 16 & 25 & 17 \\
\hline $45-55$ & M edium & 33 & 35 & 38 & 37 \\
\hline $55-65$ & Strong & 23 & 23 & 30 & 25 \\
\hline$<65$ & Critical & 29 & 26 & 7 & 21 \\
\hline
\end{tabular}

According to the data of long-term phenological observations, the vegetation leafing period in the Moscow region is about 6 months, i.e. this is the duration from full disclosure of the foliage of the arboreal layer of vegetation to the period of leaf fall. The period of established snow cover lasts on average 4 months (about 30\%), and, accordingly, about 2 months (about 20\%) the off-season period lasts.

The calculation of the average annual values of the noise level was carried out taking into account the weight coefficients of each season:

$$
\text { Noise }_{a v}=0,3 \cdot \text { Noise }_{\text {winter }}+0,2 \cdot \text { Noise }_{\text {spring-autumn }}+0,5 \cdot \text { Nois }_{\text {summer }}
$$

where, Noise $_{a v}$ - is the average annual noise level; Noise winter, Noise $_{\text {spring-autumn, Noise }}$ summer - are the values of the noise level in winter, spring-autumn, and summer seasons, respectively.

The spatial distribution of the calculated values is shown in figure 1.
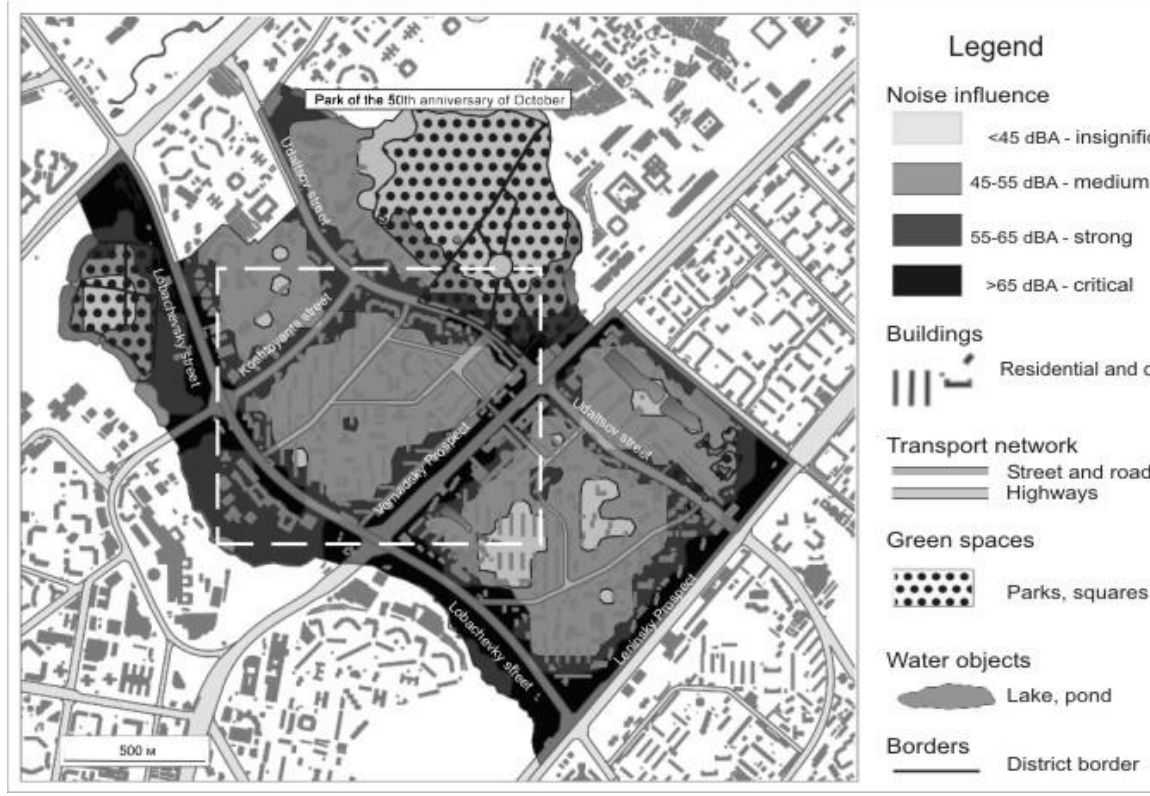

Fig. 1. Spatial distribution of the noise influence on the territory of the V ernadsky Prospect area (white dotted lines mark the boundaries of the fragment shown in fig.2.) 
Peculiarities of noise distribution over the territory of the administrative district are primarily due to the linear shape of the impact sources - highways. Thus, the area of distribution of the greatest values has an elongated shape along highways, with a thickening near the intersections of 6-8-lane highways. From the zone of critical noise levels load, noise "spreads" over the nearby territories and "flows" into the courtyard areas.At the same time, the buildings located in the first row from the highways act as noise protection barriers, behind which the process of free "spreading" is disturbed, leading to a significant drop in the noise level within the quarters. In the zone of five-storey residential buildings, local spots of reduced values are noted, which corresponds to "insignificant" and "medium" noise load. This impact is associated with the peculiarities of the urban planning of residential buildings, as well as with the noise-protective functions of trees and shrubs, which traditionally fill the inter-house territory, completely hiding buildings under their crowns [2]. In contrast to the five-storey buildings, the territory with modern urban planning (completed after 2000) does not have such an impact. In these areas, the level of the average annual noise load is recorded as "high".

Spatio-temporal studies of the obtained seasonal data revealed the dynamics of the noise level in the study area. Figure 2 shows enlarged fragments of the maps of noise level distribution by seasons (the border of the fragment is represented by a white dotted line in figure 1). As can be seen from the given fragments of the study area, the Spatio-temporal dynamics of the noise level has significant changes between seasons, with the highest values in the autumn-spring and the smallest in the summer season.

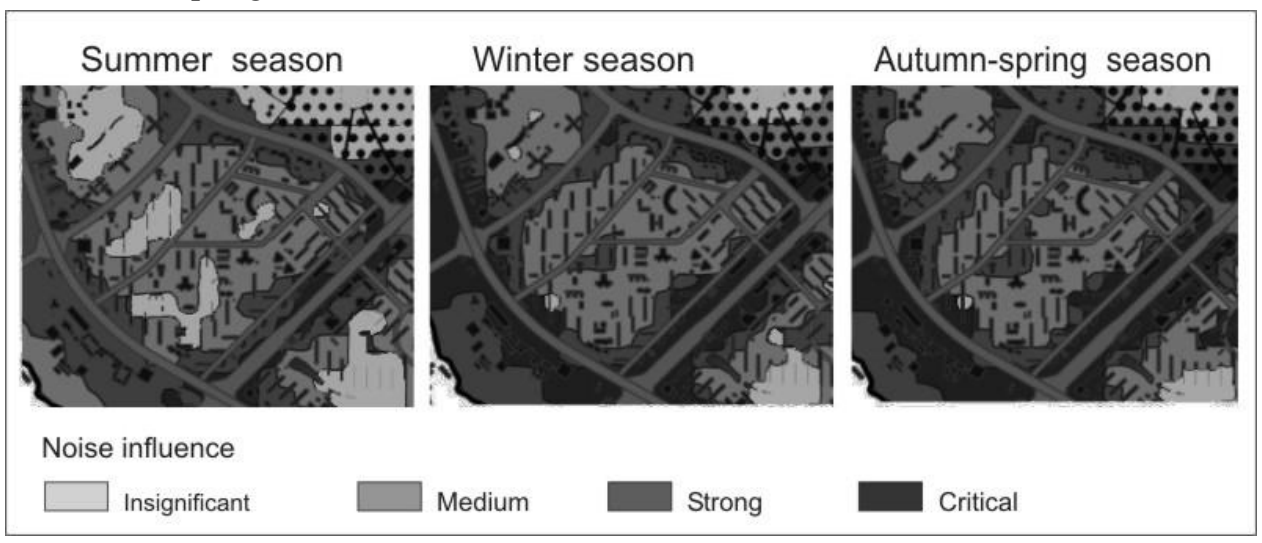

Fig. 2. Seasonal changes in noise influence (map fragments).

\section{Conclusion}

The research results show that in the annual dynamics of the noise level in urban areas there is a fierce seasonal character with the maximum level of impact in the autumn-spring period.

The highest values of the noise level are recorded along large highways, spreading inside residential areas to the first row of buildings and structures. In green areas, the noise impact penetrates much further than in the built-up area. The quarters of the five-storey housing stock form a kind of "oases" with reduced noise levels.

For the Vernadsky Prospect administrative district the territory with average annual values exceeding the permissible standards, it is $54 \%$. At the same time, the highest values of the noise level (critical level of noise influence) are recorded throughout the year along the highways and their intersections - Leninsky Prospekt - Lobachevsky Street - Vernadsky Prospect - Michurinsky Prospect. At the same time, the quarters of the five-storey residential 
building due to their planning characteristics, and due to the complete closure with vegetation, have the least level of noise influence.

There are three seasons in the annual dynamics, among which the lowest noise influence were recorded in summer (an insignificant noise level impact is $25 \%$, critical - $7 \%$ ), and the largest in autumn-spring (insignificant - 15\%, critical - 29\%). The average annual values of the share of the territory not exceeding the PEL in terms of noise influence is $54 \%$. Since a significant part of the territory with relatively low levels of impact belongs to the green zone and quarters of five-storey housing stock (with a low population density), it can be argued that the largest part of the population of the district experiences an increased noise level impact throughout the year.

\section{References}

1. Haibo W ang, M ing Cai, $\mathrm{Y}$ ifan $\mathrm{Y}$ ao, A modified $3 D$ algorithm for road traffic noise attenuation calculations in large urban areas // J ournal of Environmental M anagement, \# 196 (2017)

2. I.L. M argolina, D.S. V eselov, M .I. Ivantsova, K.A. Chavel, Features of vertical changes in the noise level in the urban area // Ecological systems and devices. No. 2 (2019)

3. Sanitary standards: SN 2.2.4/2.1.8.562-96 Noise at workplaces, in residential, public buildings and on the territory of residential buildings. M oscow (1996)

4. T.A. V orobiyova, A.V . K rasnushkin, A .A. Potapov, Study and mapping of physical pollution of the urban environment // Bulletin of M oscow University. Series 5: Geography, N 0.4 (2005)

5. Atlas of the USSR. M ain Directorate of Geodesy and Cartography, M oscow (1983) 